\title{
The Drosophila Anion Exchanger (DAE) lacks a detectable interaction with the spectrin cytoskeleton
}

\author{
Ronald R Dubreuil*1, Amlan Das², Christine Base1 and G Harper Mazock
}

\begin{abstract}
Background: Current models suggest that the spectrin cytoskeleton stabilizes interacting ion transport proteins at the plasma membrane. The human erythrocyte anion exchanger (AE1) was the first membrane transport protein found to be associated with the spectrin cytoskeleton. Here we evaluated a conserved anion exchanger from Drosophila (DAE) as a marker for studies of the downstream effects of spectrin cytoskeleton mutations.

Results: Sequence comparisons established that DAE belongs to the SLC4A1-3 subfamily of anion exchangers that includes human AE1. Striking sequence conservation was observed in the C-terminal membrane transport domain and parts of the N-terminal cytoplasmic domain, but not in the proposed ankyrin-binding site. Using an antibody raised against DAE and a recombinant transgene expressed in Drosophila S2 cells DAE was shown to be a $136 \mathrm{kd}$ plasma membrane protein. A major site of expression was found in the stomach acid-secreting region of the larval midgut. DAE codistributed with an infolded subcompartment of the basal plasma membrane of interstitial cells. However, spectrin did not codistribute with DAE at this site or in anterior midgut cells that abundantly expressed both spectrin and DAE. Ubiquitous knockdown of DAE with dsRNA eliminated antibody staining and was lethal, indicating that DAE is an essential gene product in Drosophila.

Conclusions: Based on the lack of colocalization and the lack of sequence conservation at the ankyrin-binding site, it appears that the well-characterized interaction between AE1 and the spectrin cytoskeleton in erythrocytes is not conserved in Drosophila. The results establish a pattern in which most of the known interactions between the spectrin cytoskeleton and the plasma membrane in mammals do not appear to be conserved in Drosophila.
\end{abstract}

\section{Background}

The spectrin cytoskeleton forms a submembrane protein scaffold that contributes to cell shape and membrane stability in the human erythrocyte [reviewed in [1]]. Biochemical studies identified the anion exchanger as the primary membrane anchor that attaches the spectrin cytoskeleton to the erythrocyte plasma membrane. Attachment is mediated by the protein ankyrin which serves as an adapter linking the $\mathrm{N}$-terminal cytoplasmic domain of the anion exchanger to the $\beta$ subunit of erythrocyte spectrin [2].

Subsequent studies of the spectrin cytoskeleton in more complex cells have uncovered a remarkable diver-

\footnotetext{
*Correspondence: ron@uic.edu

1 Dept. of Biological Sciences, University of Illinois at Chicago, 900 S. Ashland Ave., Chicago, IL 60607 USA

Full list of author information is available at the end of the article
}

sity of different membrane proteins attached to ankyrin. Many of these are physiologically important transporters and channels whose distribution in the cell is critical to function [3,4]. Most of these integral membrane proteins appear to rely on their interaction with the spectrin cytoskeleton to be stably expressed at the cell surface. Consequently, mutations that knock out or inactivate ankyrin or spectrin lead to a dramatic reduction in their steady-state levels.

Spectrins and ankyrins are conserved between humans and Drosophila. There is a single conventional spectrin in Drosophila that is composed of $\alpha$ and $\beta$ subunits arranged as a tetramer. Drosophila spectrin is nearly indistinguishable from human spectrin by electron microscopy, it possesses most of the known functional sites (e.g. actinbinding, ankyrin-binding, intersubunit interactions, $\mathrm{PH}$ domain, etc.) and it is found associated with the plasma 
membrane in most Drosophila cells that have been examined [5]. Ankyrin is also conserved between Drosophila and humans. Ankyrins possess an $\mathrm{N}$-terminal membrane binding domain composed of ankyrin repeats and a central spectrin binding domain. The two isoforms of ankyrin in Drosophila are similar to one another in their $\mathrm{N}$-terminal and spectrin-binding domains, but their Cterminal domains are different, with further diversity added by alternative splicing of the neuronal ankyrin isoform DAnk2 [6-8]. Interestingly, there is comparable sequence diversity between mammalian ankyrin isoforms in the C-terminal domain with only limited similarity to Drosophila ankyrins [2,7].

Yet, while spectrin and ankyrin are conserved in Drosophila, a remarkable divergence has become apparent in recent studies of candidate membrane anchors. Out of five interactions that have been examined so far only the interaction with L1 family cell adhesion molecules and ankyrin appears to be conserved in Drosophila. The L1 family member neuroglian possesses a conserved ankyrin-binding sequence in its cytoplasmic domain and it exhibits a functional interaction with ankyrin as well [9]. Another cell adhesion molecule, E-cadherin, was recently shown to interact directly with ankyrin in mammals [10]. In contrast, DE-cadherin (the fly counterpart of E-cadherin) does not appear to interact with ankyrin in Drosophila [11]. Two other ankyrin-binding membrane proteins in mammals, voltage-dependent sodium channels and KCNQ potassium channels, are conserved in their transmembrane ion-conducting domains but the domains responsible for binding to ankyrin in humans are not conserved in Drosophila [12]. The Na, K ATPase appears to be functionally linked to spectrin in Drosophila, since its behavior is altered in $\beta$ spectrin mutants [13]. However, while the connection appears to be mediated by ankyrin in mammals [14], ankyrin-binding activity does not appear to be required for the effect of spectrin on the Na, K ATPase in Drosophila [15].

To expand the repertoire of membrane proteins that can be analyzed, we took advantage of molecular tools generated by the Drosophila genome project. A homolog of the erythrocyte anion exchanger was identified in the genomic sequence of Drosophila. It was an attractive candidate for further analysis because of its well-known interaction with ankyrin in mammals. The anion exchanger belongs to a family of closely related genes (AE1, AE2 and AE3; also known as SLC4A1-3) and to a larger family of 10 related genes that transport bicarbonate (SLC4 A1-10 [ref. [16,17]]). A conserved bicarbonate transporter (NDAE1) was previously identified in Drosophila and was shown to resemble the human sodiumdependent anion exchanger SLC4A8 [18]. Here we describe the properties of a second Drosophila anion exchanger (DAE) that it is closely related to human
SLC4A1-3. Based on sequence comparisons and protein localization experiments, it appears that the well-characterized interaction between AE1 and the spectrin cytoskeleton in human erythrocytes is not conserved in Drosophila.

\section{Results}

\section{Amino acid sequence analysis}

A Drosophila anion exchanger (DAE) related to mammalian AE1 was first identified among expressed sequence tags (ESTs) from the Drosophila genome project [19]. Analysis of data in FlyBase [20] indicates that there are 6 major polypeptide classes, shown relative to the longest class (A) in Figure 1, which are encoded by a number of different mRNAs. Classes $\mathrm{E}$ and $\mathrm{D}$ use an alternate $5^{\prime}$ exon and an internal start methionine relative to $\mathrm{A}$. Classes $\mathrm{K}$ and J use an alternate splice acceptor site, leading to deletion of 35 codons. Classes B, D and J splice out an alternate exon, leading to deletion of 67 codons. We used the amino acid sequence of clone RE39419 ([ref. [21]]; class B) for all of the work reported here. The sequence differences between classes in the $\mathrm{N}$-terminal coding region occur within a region that is poorly conserved among $A E$ family members. Likewise, the sequence of the skipped exon, absent from class B, did not match the sequence of other known anion exchangers.

Sequence comparisons established that the Drosophila anion exchanger belongs to the $\mathrm{AE}$ subfamily that includes mammalian AE1, AE2 and AE3 (Table 1). This subfamily represents the $\mathrm{Na}^{+}$-independent, electro-neutral anion exchangers. DAE shares $43-45 \%$ sequence

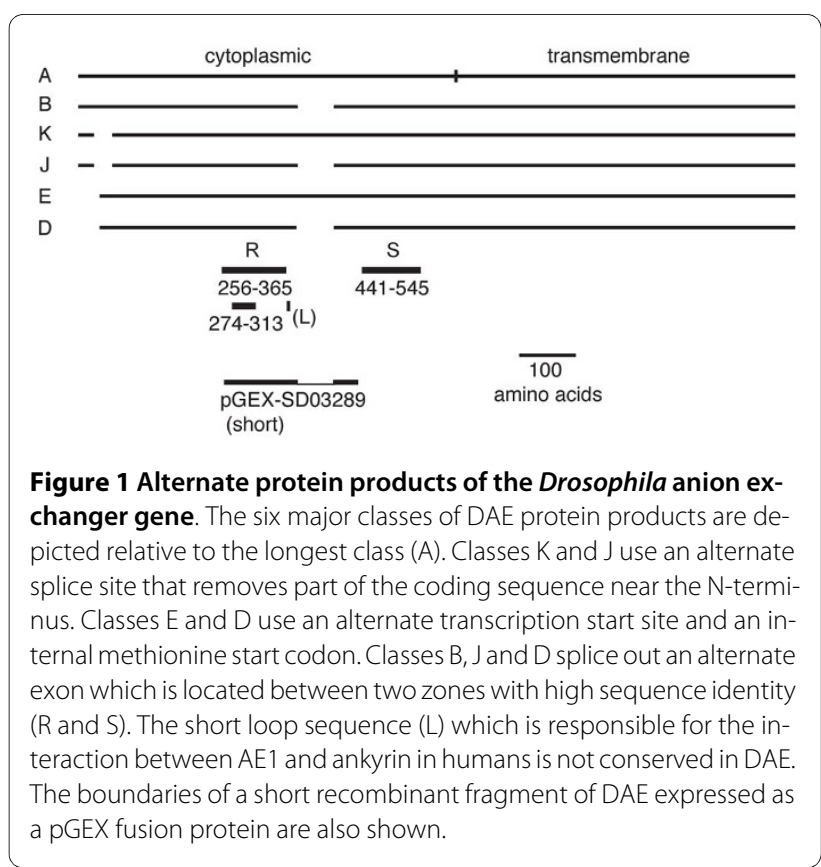


Table 1: Amino acid sequence comparison of human and Drosophila anion exchangers (\% identity)

\begin{tabular}{llllll}
\hline & NDAE1 & DAE & AE1 & AE2 & AE3 \\
\hline NDAE1 & & & & \\
DAE & - & 40 & 38 & 40 & 40 \\
AE1 & - & 45 & 43 & 44 \\
AE2 & & - & 60 & 57 \\
AE3 & & & - & 62 \\
\hline
\end{tabular}

identity with human AE1, 2 and 3, (Table 1) but only 26 $40 \%$ identity with the other 7 members of the SLC4A gene family (not shown), including NDAE1. Thus DAE and NDAE1 appear to be in distinct anion exchanger subfamilies.

Sequence alignments highlighted a number of features of the DAE sequence. First, the overall domain structure of DAE is essentially identical to human erythrocyte AE1 (Figure 2). A large $\mathrm{N}$-terminal domain, ending with amino acid 667 of DAE, corresponds to the large N-terminal cytoplasmic domain of AE1. From that point on there is a close register between the sequences of human $\mathrm{AE} 1$ and DAE, corresponding to the position of transmembrane sequences (highlighted in green) and intraand extra-cellular loops [22]. The only length variations occur in the two large extracellular loops between transmembrane domains 5 and 6 [TM5-6] and between [TM78]. The distribution of predicted glycosylation sites was not conserved between human AE1 and DAE. There are 4 N-linked glycosylation consensus sites $(\mathrm{NxS} / \mathrm{T})$ in the [TM5-6] linker of DAE (indicated by "g"), but none in human AE1 (Figure 2). There is a single conserved glycosylation site in the [TM7-8] linker of AE1 ("g"), but none in DAE. Like DAE, human AE2 had no consensus glycosylation sites in the [TM7-8] linker, one consensus site was shared with DAE in linker [TM5-6] and there were two other consensus sites in that linker that were not found in DAE. Thus there was a greater correspondence in predicted glycosylation patterns between AE2 and DAE than between AE2 and AE1.

Sequence comparisons also revealed several notable features of the $\mathrm{N}$-terminal and $\mathrm{C}$-terminal cytoplasmic domains of DAE. Two regions of substantial sequence identity near the $\mathrm{N}$-terminus ( $\mathrm{R}$ and $\mathrm{S}$, highlighted in red in Figure 2) were sandwiched between three domains of limited sequence identity. Some of this sequence conservation coincides with the cytoplasmic domain $\mathrm{pH}$ sensor of mammalian AE2 (region R [ref. [17]]). Sequence comparisons in this region established a hierarchy of sequence identities with the highest between human AE2 and AE3, the next highest between DAE and AE2/3, and the lowest between AE1 and the others. A 40 residue sequence within the first conserved region ( $R$ '; bold let- ters) has been noted previously for its conservation among anion exchangers $[17,23]$ and for its role in $\mathrm{pH}$ regulation of AE2 and AE3, but not AE1 [24]. The R' sequence was nearly identical between AE2 and AE3, DAE was $80 \%$ identical to AE2 in this region, and AE1 was $53 \%$ identical to AE2. The other conserved region (S) exhibited the same overall pattern of sequence identities, further demonstrating divergence of AE1 relative to DAE.

Recent structural studies mapped an ankyrin interaction site within an 11 amino acid loop in the $\mathrm{N}$-terminal cytoplasmic domain of AE1 (L in Figure 1; bold black characters in Figure 2 [ref. [25]]). That loop falls in between the zones of sequence identity described above. In fact, the sequence alignment between DAE and AE1 inserted a gap precisely at that site, because of the limited sequence homology between the two proteins in between regions $\mathrm{A}$ and $\mathrm{B}$. Gaps were also introduced at this site in comparisons between human AE1, AE2 and AE3, suggesting that this binding site is not a conserved feature of the AE gene family.

The sequence LDADD near the C-terminus of AE1 (purple text) is thought to be responsible for a functional interaction between carbonic anhydrase and mammalian anion exchangers [26]. A similar sequence was present in AE2 (LDANE), AE3 (LDSED), and in DAE (LDGSE), but not in NDAE1 (LDDIM). This pattern of sequence conservation further supports the grouping of DAE within the AE subfamily of anion exchangers.

\section{Production and characterization of a DAE antibody}

A polyclonal antiserum was produced in rabbits using a purified glutathione transferase fusion protein containing 140 amino acids from the cytoplasmic domain of DAE. The resulting antibody produced a robust response in western blots of the recombinant fusion protein (not shown). The antibody was affinity-purified before further use and cross-adsorbed with purified glutathione transferase.

We engineered a recombinant DAE transgene carrying a myc epitope tag at the $\mathrm{N}$-terminus of the protein. The coding sequence of cDNA RE39419 was used to produce the construct. The purified anti-DAE antibody was used to stain western blots of total proteins from Drosophila 


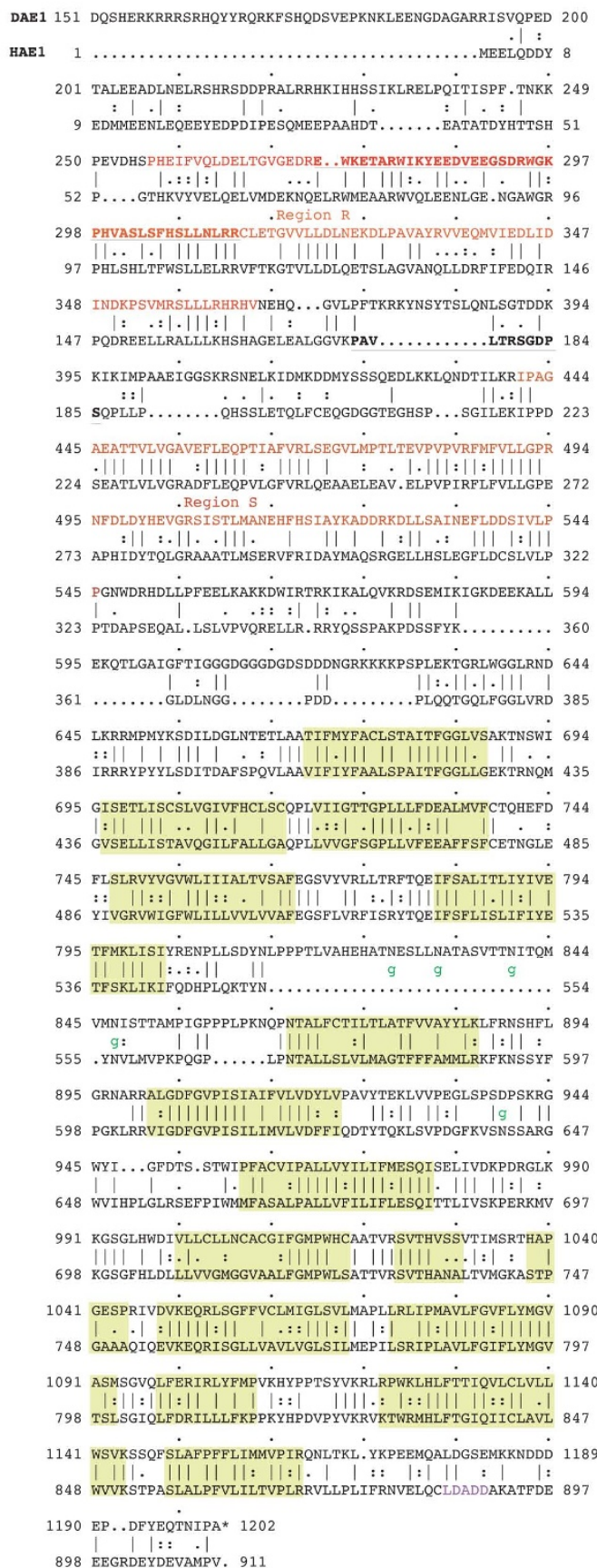

Figure 2 Amino acid sequence alignment between DAE and human erythrocyte AE1. The positions of 16 predicted transmembrane sequences are indicated in green boxes. The boundaries of the conserved cytoplasmic domain sequences $\mathrm{R}$ and $\mathrm{S}$ are indicated in red. The conserved subregion $R^{\prime}$ is indicated in bold red type. The sequence of the ankyrin-binding site in human AE1 is indicated in bold black type. Consensus glycosylation sites in the linker between transmembrane regions $[5,6]$ and $[7,8]$ are each marked by $\mathrm{g}$. The site of interaction between the $\mathrm{C}$-terminal domain of $\mathrm{AE} 1$ and carbonic anhydrase is indicated in purple.
S2 tissue culture cells. Reactions with control S2 cells detected a faint band with the expected mobility of fulllength DAE ( 136 kD; Figure 3, lane 4). The relative intensity of the band increased in transfected cells transiently expressing recombinant DAE (lane 5). The same size band was detected with the myc-epitope antibody in transfected cells (lane 3) but not in non-transfected controls (lane 1). A control reaction with transfected cells expressing myc-tagged $\beta$ spectrin detected a distinct 278 $\mathrm{kD}$ band (lane 2).

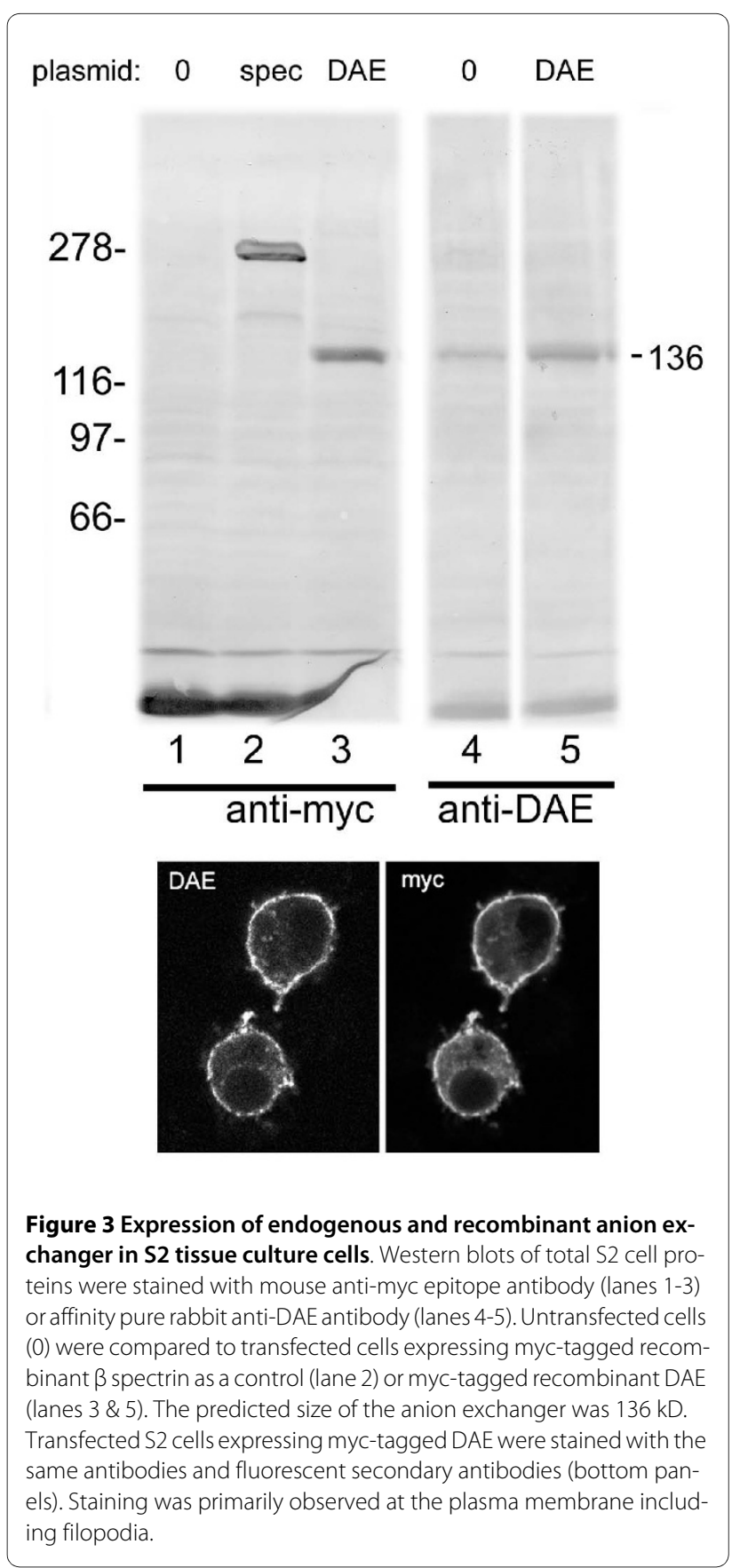


The same antibodies were used for immunofluorescent staining of S2 cells expressing recombinant myc-tagged DAE. Staining of control cells with the DAE antibody produced very faint plasma membrane staining, close to the threshold of detection (not shown). However, both the myc tag and the DAE antibodies produced strong plasma membrane staining in transfected cells expressing recombinant DAE (Figure $3 \mathrm{~B}$ ). These results establish that the affinity purified DAE antibody detects the expected protein product and that DAE is a plasma membrane protein.

\section{Localization of DAE in larval tissues}

The first issue we wished to address with the DAE antibody was its staining pattern in the larval digestive tract, so that we could evaluate its potential involvement in the stomach acid secretion phenotype of $\alpha$ spectrin mutants [27]. Fortuitously, the most prominent region of staining that we observed in larvae was in the midgut (Figure 4A). Within the midgut (Figure 4A), the DAE antibody brightly stained the copper cell region (CC), a cell cluster anterior to the copper cells (AC), the large flat cells (LFC), and a more posterior cluster of $2-3$ cells $(\mathrm{PC})$ that may correspond to the iron cells (Figure 4A; [28]). In the anterior cells, DAE staining was confined to the basal surface of the plasma membrane (Figure $4 \mathrm{~A} \& 4 \mathrm{H}$ ). In contrast, $\alpha$ spectrin was abundant at lateral sites of cell-cell contact as well as at the basal plasma membrane (Figure 4G). Faint apical staining corresponds to the $a \beta_{\mathrm{H}}$ isoform of spectrin [29]. The large flat cells (LFC) and posterior cells (PC) have an extremely flat morphology that makes it difficult to judge which membrane domain(s) were labeled by the antibody. Staining in the latter two zones was usually limited to cells on only one side of the epithelial tube.

Closer inspection revealed that DAE staining in the copper cell region did not correspond to the copper cells themselves (Figure 4B). Instead, the copper cells were identifiable by their lack of staining with the DAE antibody, and by the bright staining of their apical and basolateral plasma membrane domains with the anti- $\alpha$ spectrin antibody (Figure 4C; merged in D). The DAE signal came from the spool-shaped interstitial cells found in between the copper cells in the middle midgut. Within the interstitial cells, DAE staining formed a gradient that was brightest at the basal surface of the cell and then diminished as it approached the perinuclear cytoplasm near the cell apex. This pattern corresponds to the elaborate infoldings of the basal plasma membrane that are seen by electron microscopy (not shown; [28]), which are most dense in the basal region region of the cell but in

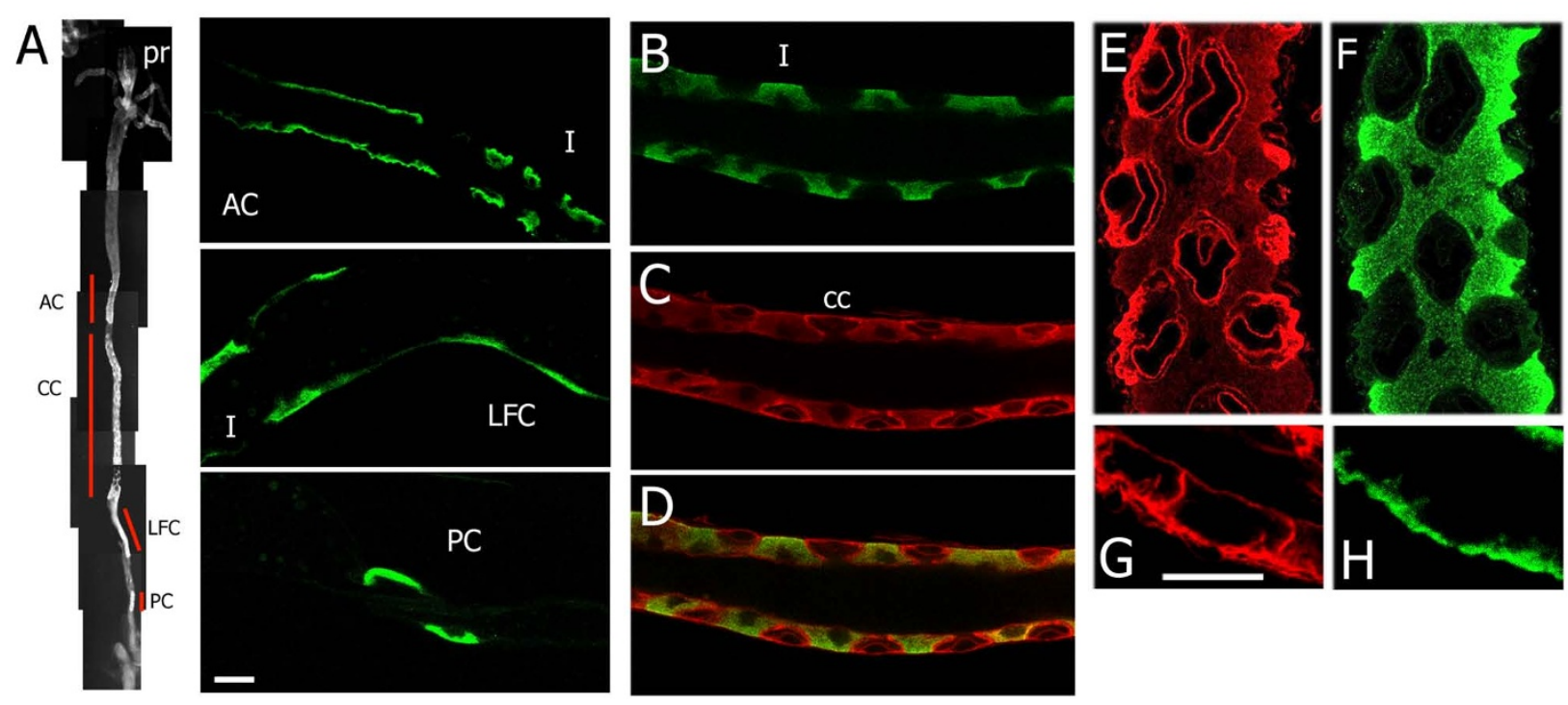

Figure 4 Immunolocalization of DAE in the larval midgut. A) Four distinct domains of DAE antibody-labeled cells in larval midgut: anterior cells (AC) upstream of the copper cell domain (CC), large flat cells (LFC) immediately downstream of the CC domain, and 2-3 posterior cells (PC) downstream of the LFC ( $\mathrm{Pr}$ = proventriculus). The higher magnification views (bar = $10 \mathrm{um}$ ) show continuous labeling of the basal membrane of adjacent cells in the AC region (vs. interstitial cells (I) that alternate with copper cells downstream). LFCs were usually visible on only one side of the epithelial tube, downstream of the last interstitial cell. B) DAE staining in the copper cell region revealed a pattern of labeled interstitial cells (I) separated by unlabeled copper cells. C) The copper cells stained with anti-a spectrin antibody appeared as lozenge shapes with relatively bright staining of the basolateral region (merged in D). Higher magnification views of the a spectrin (E) and DAE (F) staining patterns emphasize their lack of overlap. Alpha spectrin was most conspicuous in the basolateral zone of contact between copper cells and interstitial cells and in the banana-shaped apical invagination of copper cells. In contrast, DAE was most conspicuous within the basal cytoplasm of interstitial cells and extended apically in a gradient. G\&H) High magnification views of the anterior cells stained for a spectrin $(G)$ or DAE $(H)$ reveals their overlapping distribution in the basal membrane region, but not in the lateral region of cell-cell contact (Bar $=10$ um) 
some cases extend nearly to the apical surface. A higher magnification view comparing the distribution of DAE (Figure $4 \mathrm{~F}$ ) to $\alpha$ spectrin $(\mathrm{E})$ revealed that $\alpha$ spectrin staining was largely confined to sites of contact between copper cells and interstitial cells and to the bananashaped apical invagination of the copper cell plasma membrane. The gradient of DAE staining in interstitial cells (Figure 4F) had no counterpart in the $\alpha$ spectrin staining pattern $(\mathrm{E})$.

A control for antibody staining was performed using a UAS-RNAi fly line from the Vienna Drosophila RNAi Center (VDRC [ref. [30]]) targeted against DAE. Knockdown of DAE expression was achieved by crossing heterozygous UAS-RNAi parents (UAS-39492/TM3GFP) to Mex-Gal4, a homozygous line that expresses Gal4 in the larval midgut [31]. Two classes of larval progeny were obtained: a $\mathrm{GFP}^{+}$control class, where GFP expression indicates the absence of the RNAi-encoding transgene, and a GFP- class that expresses RNAi. Staining of these larvae with anti-DAE antibody revealed a striking reduction in DAE antibody staining in interstitial cells (Figure 5D) relative to the normal pattern in controls (Figure 5A). The midgut DAE knockdown had no detectable effect on larva viability and there was no detectable effect on midgut acidification (not shown) as detected by Bromphenol blue feeding [27]. However, ubiquitous expression of DAE RNAi using tubP-Gal4 [32] produced a lethal phenotype, indicating that there is a critical requirement for DAE function elsewhere in the animal. The lethal RNAi phenotype is consistent with the recessive lethality of a transposable element insertion in the DAE gene that was recently produced by the Berkeley
Drosophila Genome Project (p[WhY]CG8177DG29506; Flybase).

\section{Discussion}

We identified and partially characterized a close relative of the vertebrate SLC4 anion exchangers in Drosophila and named it DAE. The amino acid sequence of this protein shares many of the characteristics of other members of this protein family, suggesting that it is likely to mediate $\mathrm{Na}$-independent anion exchange in vivo. One major site of expression identified in this study is the stomach acid-secreting region of the larval midgut. We anticipate that there are other important sites of expression given that RNAi-mediated knockdown of DAE expression in the midgut was not lethal whereas ubiquitous knockdown of DAE with RNAi was lethal. Independent confirmation of the essential function of DAE comes from the recent identification of a recessive lethal transposable element insertion in the DAE gene (Flybase).

We previously speculated that a defect in anion exchange activity could account for the stomach acid secretion defect in Drosophila a spectrin mutants [27]. This prediction was based on the known interaction of mammalian anion exchangers with ankyrin (and hence spectrin) and the known contribution of anion exchange to acid secretion in mammals. Targeted disruption of mouse AE2 clearly demonstrated an essential role in gastric acid secretion [33]. Deletion of another anion exchanger gene family member (Slc26a9) also blocked gastric acid secretion in the mouse because of its likely effect on chloride secretion [34]. Yet, a knockdown of DAE that was sufficient to eliminate detectable immuno-
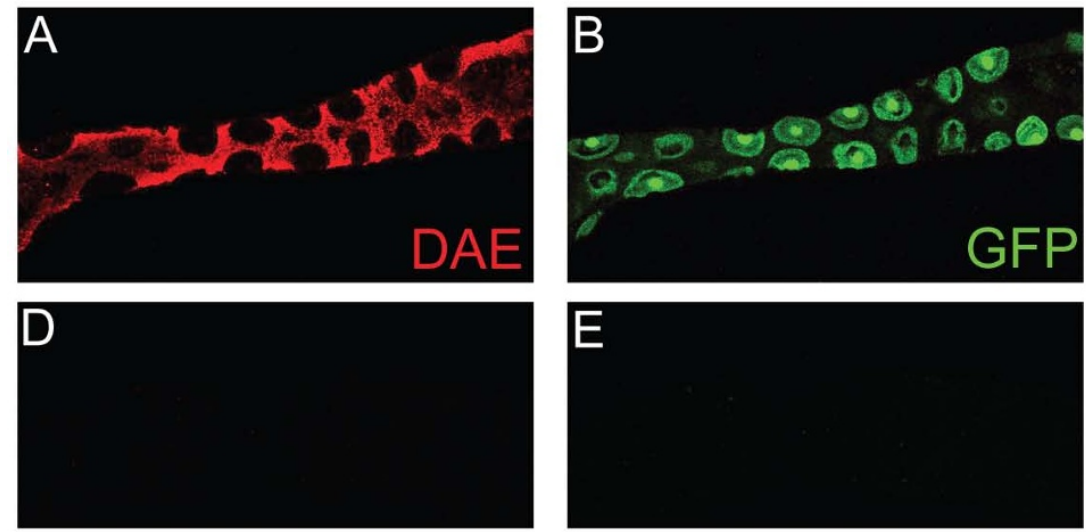
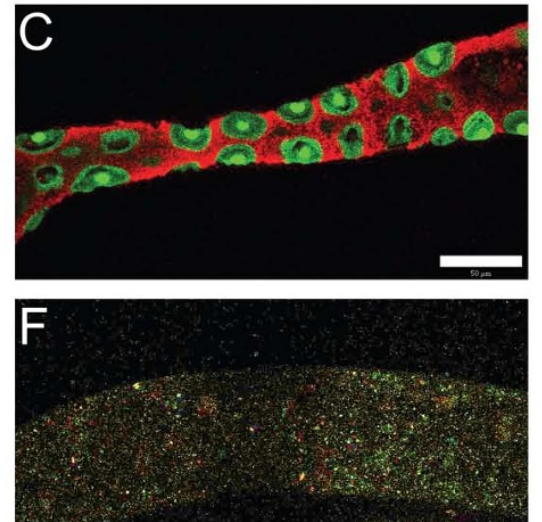

Figure $\mathbf{5}$ RNAi knockdown of DAE expression. The specificity of the DAE antibody was tested by knocking down its expression with RNAi. The midgut-specific Mex-Gal4 driver was used to induce RNAi. In the cross scheme used RNAi-expressing larvae were distinguished from non-expressing siblings by the presence of a GFP reporter in the latter. Larvae were sorted by GFP expression and then dissected and stained with the anti-DAE antibody followed by Texas Red labeled secondary antibody. Control larvae carrying the GFP-marked balancer chromosome exhibited the expected pattern of interstitial cell DAE staining (A) with the GFP reporter fortuitously expressed in neighboring copper cells (B). Siblings that expressed UAS-RNAi (recognized by lack of GFP; E) showed no detectable DAE staining (D), indicating that the antibody was specific for DAE. The merged image (F) was overexposed to demonstrate the presence of the middle midgut. Bar $=20 \mathrm{um}$. 
reactivity had no detectable effect on the ability of larvae to produce stomach acid. Thus a different downstream target is likely to be responsible for the observed acid secretion defect in $\alpha$ spectrin mutants.

We set out to characterize DAE with the intent of using it as a membrane marker for the effects of spectrin mutations on interacting membrane proteins. Human erythrocyte AE1 is the best known membrane anchor for ankyrin and spectrin [1]. We conclude from the present evidence that DAE is unlikely to interact with the spectrin cytoskeleton in Drosophila. Mammalian membrane proteins that interact with the spectrin cytoskeleton in vivo typically colocalize with spectrin and ankyrin by immunofluorescence. Using a sensitive antibody that readily detects spectrin in most Drosophila cells, we find that little or no spectrin is expressed in the interstitial cells where DAE is abundantly expressed. If spectrin is present and codistributes with DAE in interstitial cells it is below the threshold of detection of this antibody. Thus, we propose that a spectrin-independent mechanism is likely to explain the peculiar polarized distribution of DAE within basal invaginations of the interstitial cell plasma membrane. In mammalian MDCK and HBE cells, spectrin, ankyrin, the Na, K ATPase, and E-cadherin form a molecular complex and codistribute along lateral sites of cellcell contact in these polarized epithelial cells $[10,35,36]$. However, in the anterior cells of the Drosophila midgut (AC), in which spectrin and DAE were both expressed, there was no detectable colocalization of the two proteins along lateral contacts between neighboring cells.

Amino acid sequence comparisons also failed to detect conservation of ankyrin-binding activity in DAE. The ankyrin binding site of human erythrocyte AE1 has been mapped to a loop within the $\mathrm{N}$-terminal cytoplasmic domain [25]. Yet, while there was remarkable amino sequence conservation among anion exchangers in regions flanking this loop. (regions R \& S in Figure 2), the sequence of the ankyrin-binding loop itself was not conserved in DAE. The flanking sequence conservation is believed to reflect a pH sensing mechanism [24,37], and is not thought to be related to ankyrin-binding activity. There is limited evidence suggesting an interaction between ankyrin and human AE2 and AE3 [38,39]. But sequence comparisons failed to detect conservation of the ankyrin-binding site in these molecules either (not shown). Thus the ankyrin-binding sequence of AE1 may be a unique byproduct of erythrocyte evolution.

The apparent lack of an interaction between DAE and the spectrin cytoskeleton matches a pattern that has emerged in a number of recent studies. As described in the introduction, there are other membrane proteins with ankyrin-binding activity in mammals that do not appear to be conserved in Drosophila. We now add DAE to the list, leaving the L1 family cell adhesion molecule neuro- glian as the only ankyrin-binding membrane protein whose interaction with ankyrin can be detected in Drosophila. What does this apparent lack of conservation mean? One possibility is that many of the known membrane interactions with the spectrin cytoskeleton arose through a physiological need that emerged in the course of vertebrate evolution. Thus, perhaps sodium channels and potassium channels do not require anchorage to the cytoskeleton to carry out their functions in Drosophila. Alternatively, it is possible that interactions between the spectrin cytoskeleton and integral membrane proteins are transient in evolution. Perhaps functional links can be swapped between different scaffold proteins such that membrane transporters in Drosophila are now linked to cytoskeletal scaffold proteins other than spectrin and ankyrin. Indeed, Drosophila spectrin function appears to be redundant in many of the cells that express it (manuscript in preparation), which may be conducive to rapid evolution of protein interactions. If so, it may turn out that different casts of membrane characters are associated with the spectrin cytoskeleton in Drosophila and mammals. Further insights into these issues are likely to emerge as the membrane anchors that attach the spectrin cytoskeleton to the plasma membrane in Drosophila are discovered and characterized.

\section{Methods}

\section{Cloning and DNA sequencing}

The full-length cDNA RE39419 was obtained from the Drosophila Genomics Resource Center. We extended the partial cDNA sequence data available in Flybase to establish that RE39419 belongs to mRNA class B (Figure 1). All sequence analysis was carried out in the DNA services facility at the University of Illinois at Chicago Research Resource Center. All oligonucleotide primers were obtained from Operon. DNA and protein sequence analysis was carried out using GCG software [40]. Amino acid sequence alignments were created using the Gap program. The human anion exchanger accession numbers used were AE1 (X12609), AE2 (NM_003040), and AE3 (NM_005070).

A 420 bp BglII - EagI fragment from the cytoplasmic domain coding region was cloned in the pGEX-3X vector[41] for expression in E coli DH5 $\alpha$. Expression studies in Drosophila S2 tissue culture cells were carried out using the pWUMB vector [13] which uses the Drosophila ubiquitin promoter to drive expression of an $\mathrm{N}$-terminally myc-tagged product. A near-full-length BamHI fragment of the RE39419 cDNA clone was used. An internal BamHI site was inactivated by QuickChange mutagenesis to produce a silent mutation in the coding sequence. Both expression constructs were verified by DNA sequencing of the cloning junctions. 


\section{Production of DAE antibody}

Expression of the glutathione transferase fusion protein with the cytoplasmic fragment of DAE was induced by IPTG and the protein was purified by affinity chromatography. Rabbits were immunized by popliteal lymph node injection of 50 ug purified antigen, followed by 50 ug antigen subdermally at one month intervals. Rabbits were pre-screened for lack of preimmune reactivity and they showed a marked response to the antigen by the second boost (not shown). Immune serum was affinity purified and antibody was counter-adsorbed with purified glutathione transferase for all of the studies described here.

\section{Expression in S2 cells, western blots, and confocal microscopy}

Transfection of S2 cells was carried out using lipofectamine (Invitrogen). Cells were processed for staining as previously described [9] using mouse monoclonal 9E10 (anti-myc; [ref. [42]]) and the affinity purified rabbit anti-DAE followed by Texas Red anti-mouse and FITC anti-rabbit secondary antibodies (Zymed Laboratories). Western blots were produced using standard methods, stained using the above antibodies with alkaline phosphatase-coupled secondary, and developed using BCIP and NBT (also from Zymed). Stained cells were viewed and photographed using an Olympus FV500 confocal microscope using a $60 \times$ plan-apo oil-immersion objective (N.A. 1.4) and Fluoview 2.1 software. Brightness settings were optimized for saturation using the photomultiplier and settings were kept constant in comparisons of transfected and untransfected cells.

\section{Competing interests}

The authors declare that they have no competing interests.

\section{Authors' contributions}

RRD conceived the study, designed the experiments, helped with data acquisition and analysis and wrote the manuscript. All authors read and approved the manuscript.

AD performed transfections, produced full length expression plasmid and performed localization experiments.

CB produced bacterial expression constructs and produced the antibody and performed DNA sequence analysis.

GHM performed RNAi experiment and localization of DAE.

\section{Acknowledgements}

We thank Srilakshmi Dhulipala for technical assistance. Supported by NIH GM49301 to RRD.

\section{Author Details \\ ${ }^{1}$ Dept. of Biological Sciences, University of Illinois at Chicago, 900 S. Ashland Ave., Chicago, IL 60607 USA and 2433 S. University Ave., Lynch Laboratories, Dept. of Biology, University of Pennsylvania, Philadelphia, PA 19104 USA}

Received: 15 March 2010 Accepted: 23 June 2010

Published: 23 June 2010

\section{References}

1. Lux SE, Palek J: Disorders of the Red Cell Membrane. In Blood: Principles and practice of hematology Edited by: Handin RI, Lux SE, Stossel TP. Philadelphia: J.B. Lippincott Co.; 1995:1701-1818.
2. Bennett $V$, Baines AJ: Spectrin and ankyrin-based pathways: Metazoan inventions for integrating cells into tissues. Physiol Rev 2001:1353-1388.

3. Dubreuil RR: Functional links between membrane transport and the spectrin cytoskeleton. J Membrane Biology 2006, 211:151-161.

4. Bennett $\mathrm{V}$, Healy J: Organizing the fluid membrane bilayer: diseases linked to spectrin and ankyrin. Trends in Molecular Medicine 2008, 14:28-36.

5. Dubreuil RR: Spectrin function: A survey of genetic systems from Drosophila to humans. In Advance in Molecular Cell Biology Volume 37 Edited by: Khurana S. New York:Elsevier; 2006:68-88.

6. Dubreuil RR, Yu J: Ankyrin and $\beta$ spectrin accumulate independently of a spectrin in Drosophila. Proc Natl Acad Sci USA 1994, 91:10285-10289.

7. Koch I, Schwarz H, Beuchle D, Goellner B, Langegger M, Aberle H: Drosophila ankyrin 2 is required for synaptic stability. Neuron 58:210-222.

8. Pielage J, Cheng L, Fetter R, Carlton PM, Sedat JW, Davis GW: A presynaptic giant ankyrin stabilizes the NMJ through regulation of presynaptic microtubules and transsynaptic cell adhesion. Neuron 2008, 58:195-209.

9. Dubreuil RR, MacVicar GR, Dissanayake S, Liu C, Homer D, Hortsch M: Neuroglian-mediated adhesion induces assembly of the membrane skeleton at cell contact sites. J Cell Biol 1996, 133:647-655.

10. Kizhatil K, Davis JQ, Davis L, Hoffman J, Hogan BLM, Bennett V: Ankyrin-G is a molecular partner of E-cadherin in epithelial cells and early embryos. J Biol Chem 2007, 282:26552-26561.

11. Dubreuil RR, Grushko T: Neuroglian and DE-cadherin activate independent cytoskeleton assembly pathways in Drosophila S2 cells. Biochem Biophys Res Commun 1999, 265:372-375.

12. Pan Z, Kao T, Lemos ZHJ, Sul J-Y, Cranstoun SD, Bennett V, Scherer SS, Cooper EC: A common ankyrin-G-based mechanism retains KCNQ and Nav channels at electrically active domains of the axon. J Neurosci 2006, 26:2599-2613.

13. Dubreuil RR, Wang P, DahI SC, Lee JK, Goldstein LSB: Drosophila beta spectrin functions independently of alpha spectrin to polarized the $\mathrm{Na}$, K ATPase in epithelial cells. J Cell Biol 2000, 149:647-656.

14. Nelson WJ, Veshnock PJ: Ankyrin binding to ( $\mathrm{Na}+\& \mathrm{~K}+)$ ATPase and implications for the organization of membrane domains in polarized cells. Nature 1987, 328:533-536.

15. Das A, Base C, Dhulipala S, Dubreuil RR: Spectrin functions upstream of ankyrin in a spectrin cytoskeleton assembly pathway. J Cell Biol 2006, 175:325-335.

16. Romero MF, Fulton CM, Boron WF: The SLC4 family of HCO3transporters. Pflugers Arch-Eur J Physiol 2004, 447:495-509.

17. Alper SL: Molecular physiology of SLC4 anion exchangers. Exp Physiol 2006:153-161.

18. Romero MF, Henry D, NBelson S, Harte PJ, Dillon AK, Sciortino CM: Cloning and characterization of a Na-driven anion exchanger (NDAE1). J Biol Chem 2000, 275:24552-24559.

19. Celniker SE, Wheeler DA, Kronmiller B, Carlson JW, Halpern A, Patel S, Adams M, Champe M, Dugan SP, Frise E, Hodgson A, George RA, Hoskins RA, Laverty T, Muzny DM, Nelson CR, Pacleb JM, Park S, Pfeiffer BD, Richards S, Sodergren EJ, Svirskas R, Tabor PE, Wan K, Stapleton M, Sutton GG, Venter C, Weinstock G, Scherer SE, Myers EW, Gibbs RA, Rubin GM: Finishing a whole-genome shotgun: release 3 of the Drosophila melanogaster euchromatic genome sequence. Genome Biology 2002, 3:RESEARCH0079.

20. Grumbling G, Strelets V, Consortium F: FlyBase: anatomical data, images and queries. Nucleic Acids Research 2006, 34:D484-D488.

21. Stapleton M, Carlson J, Brokstein P, Yu C, George MCR, Guarin H, Kronmiller B, Pacleb J, Park S, Wan K, Rubin GM, Celniker SK: A Drosophila full-length cDNA resource. Genome Biology 2002, 3:research0080.

22. Zhu Q, Lee DWK, Casey JR: Novel topology in C-terminal region of the human plasma membrane anion exchanger, AE1. J Biol Chem 2003, 278:3112-3120.

23. Romero MF, Hediger MA, Boulpaep EL, Boron WF: Expression cloning and characterization of a renal electrogenic $\mathrm{Na}+\mathrm{HCO} 3$ - cotransporter. Nature 1997, 387:409-413.

24. Stewart AK, Chernova M, Shmukler BE, Wilhelm S, Alper SL: Regulation of $\mathrm{AE} 2$-mediated $\mathrm{Cl}$ - transport by intracellular or by extracellular $\mathrm{pH}$ requires highly conserved amino acid residues of the $A E 2 \mathrm{NH} 2-$ terminal cytoplasmic domain. J Gen Physiol 2002, 120:707-722. 
25. Chang SH, Low PS: Identification of a critical ankyrin-binding loop on the cytoplasmic domain of eruthrocyte membrane band 3 by crystal structure analysis and site-directed mutagenesis. J Biol Chem 2003, 278:6879-6884

26. Sterling D, Reithmeier RAF, Casey JR: A transport metabolon. J Biol Chem 2001, 276:47886-47894.

27. Dubreuil RR, Frankel J, Wang P, Howrylak J, Kappil M, Grushko T: Mutations of alpha spectrin and labial block cuprophilic cell differentiation and acid secretion in the middle midgut of Drosophila larvae. Dev Biol 1998, 194:1-11.

28. Filshie BK, Poulson DF, Waterhouse DF: Ultrastructure of the copperaccumulating region of the Drosophila larval midgut. Tissue \& Cell 1971, 3:77-102.

29. Dubreuil RR, Maddux PB, Grushko T, MacVicar GR: Segregation of two spectrin isoforms: polarized membrane binding sites direct polarized membrane skeleton assembly. Mol Biol Cell 1997, 8:1933-1942.

30. Dietzl G, Chen D, Schnorrer F, Su KC, Barinova Y, Fellner M, Gasser B, Kinsey K, Oppel S, Scheiblauer S, Couto A, Marra V, Keleman K, Dickson BJ: A genome-wide transgenic RNAi library for conditional gene inactivation in Drosophila. Nature 2007, 448:151-156.

31. Phillips $M D$, Thomas $G H$ : Brush border spectrin is required for early endosome recycling in Drosophila. J Cell Sci 2006, 119:1361-1370.

32. Lee T, Luo L: Mosaic analysis with a repressible neurotechnique cell marker for studies of gene function in neuronal morphogenesis. Neuron 1999, 22:451-461.

33. Gawenis LR, Ledoussal C, Ju LM, Prasad V, Alper SL, Stuart-Tilley A, Woo AL, Grisham C, Sanford LP, Doetschman T, Miller ML, Shull GE: Mice with a targeted disruption of the $\mathrm{AE2} 2 \mathrm{Cl}-/ \mathrm{HCO} 3$ - exchanger are achlorhydric. $J$ Biol Chem 2004, 279:330531-330539.

34. Xu J, Song P, Miller ML, Borgese F, Barone S, Riederer B, Wang Z, Alper SL, Forte JG, Shull G, Ehrenfeld J, Seidler U, Soleimani M: Deletion of the chloride trasnporter Slc26a9 causes loss of tubulovesicles in parietal cells and impairs acid secretion in the stomach. Proc Natl Acad Sci USA 2008, 105:17955-17960.

35. Nelson WJ, Hammerton RW: Identification of a membrane-cytoskeleton complex containing the cell adhesion molecule uvomorulin (ECadherin), ankyrin, and fodrin in Madin-Darby canine kidney epithelial cells. J Cell Biol 1990, 110:349-357.

36. Kizhatil $K$, Bennett $V$ : Lateral membrane biogenesis in human bronchial epithelial cells requires 190-kDa ankyrin-G. J Biol Chem 2004, 279:16706-16714.

37. Stewart AK, Chernova MN, Kunes YZ, Alper SL: Regulation of AE2 anion exchanger by intracellular $\mathrm{pH}$ : Critical regions of the $\mathrm{NH} 2$-terminal cytoplasmic domain. Am J Physiol Cell Physiol 2001, 281:C1344-1354.

38. Jons T, Drenckhahn D: Anion exchanger 2 (AE2) binds to erythrocyte ankyrin and is colocalized with ankyrin along the basolateral plasma membrane of human gastric parietal cells. E J Cell Biol 1998, 75:232-236.

39. Morgans CW, Kopito RR: Association of the brain anion exchanger, AE3, with the repeat domain of ankyrin. J Cell Science 1993, 105:1137-1142.

40. Devereux J, Haeberli P, Smithies O: A comprehensive set of sequence analysis programs for the VAX. Nucl Ac Res 1984, 12:387-395.

41. Smith DB, Johnson KS: Single-step purification of polypeptides expressed in Escherichia coli as fusions with glutathione S-transferase. Gene 1988, 67:31-40.

42. Evan Gl, Lewis GK, Ramsay G, Bishop JM: Isolation of monoclonal antibodies specific for human c-myc proto-oncogene product. Mo/ Cell Biol 1985, 5:3610-3616.

doi: 10.1186/1477-5751-9-5

Cite this article as: Dubreuil et al., The Drosophila Anion Exchanger (DAE) lacks a detectable interaction with the spectrin cytoskeleton Journal of Negative Results in BioMedicine 2010,9:5

\section{Submit your next manuscript to BioMed Central} and take full advantage of:

- Convenient online submission

- Thorough peer review

- No space constraints or color figure charges

- Immediate publication on acceptance

- Inclusion in PubMed, CAS, Scopus and Google Scholar

- Research which is freely available for redistribution

Submit your manuscript at www.biomedcentral.com/submit
C Biomed Central 